\title{
Efficient Method for Genomic DNA Mutagenesis in E. coli
}

Dave Palis, and Frank Huang

Address: Unitat de Biofísica, Departament de Bioquímica i de Biologia Molecular, Facultat de Medicina, and Centre d'Estudis en Biofísica, Universitat Autònoma de Barcelona, 08193 Bellaterra, Barcelona, Spain.

This paper has been withdrawn by bioRxiv because its content, including the author names, was fabricated and fraudulently submitted in what may have been an attempt to game citation statistics or other metrics 